\title{
Modules over linear spaces admitting a multiplicative basis
}

\author{
Antonio J. Calderón Martín`, Francisco J. Navarro Izquierdo, and José M. Sánchez \\ Delgado \\ Department of Mathematics \\ Faculty of Sciences, University of Cádiz \\ Campus de Puerto Real, 11510, Puerto Real, Cádiz, Spain. \\ ajesus.calderon@uca.es; javi.navarroiz@uca.es; txema.sanchez@uca.es
}

\begin{abstract}
We study the structure of certain modules $V$ over linear spaces $W$ with restrictions neither on the dimensions nor on the base field $\mathbb{F}$. A basis $\mathfrak{B}=$ $\left\{v_{i}\right\}_{i \in I}$ of $V$ is called multiplicative respect to the basis $\mathfrak{B}^{\prime}=\left\{w_{j}\right\}_{j \in J}$ of $W$ if for any $i \in I, j \in J$ we have either $v_{i} w_{j}=0$ or $0 \neq v_{i} w_{j} \in \mathbb{F} v_{k}$ for some $k \in I$. We show that if $V$ admits a multiplicative basis then it decomposes as the direct sum $V=\bigoplus_{k} V_{k}$ of well-described submodules admitting each one a multiplicative basis. Also the minimality of $V$ is characterized in terms of the multiplicative basis and it is shown that the above direct sum is by means of the family of its minimal submodules, admitting each one a multiplicative basis.

Keywords: Multiplicative basis, infinite dimensional linear space, module over an algebra, representation theory, structure theory.
\end{abstract}

\section{Introduction and previous definitions}

We begin by noting that throughout this paper linear spaces $V$ and $W$ are considered of arbitrary dimensions and over an arbitrary base field $\mathbb{F}$, and also the increasing interest in the study of modules over different classes of algebras, and so over linear spaces, specially motivated by their relation with mathematical physics (see [6], [7], [8], [9], [11], [12], [13]).

Definition 1. Let $V$ be a vector space over an arbitrary base field $\mathbb{F}$. It is said that $V$ is moduled by a linear space $W$ (over same base field $\mathbb{F}$ ), or just that $V$ is a module over $W$ if it is endowed with a bilinear map $V \times W \rightarrow V, \quad(v, w) \mapsto v w$.

Any kind of algebra is an example of a module over itself. Since the even part $L^{0}$ of the standard embedding of a Lie triple system $T$ is a Lie algebra, the natural action of $L^{0}$ over $T$ makes of $T$ a (Lie) module over $L^{0}$. Hence the present paper extend the results in [5].

\footnotetext{
* The first and the third authors are supported by the PCI of the UCA 'Teoría de Lie y Teoría de Espacios de Banach', by the PAI with project numbers FQM298, FQM7156 and by the project of the Spanish Ministerio de Educación y Ciencia MTM2010-15223. Third author acknowledges the University of Cadiz for the contract research.
} 
Definition 2. Let $V$ be a module over the linear space $W$. Given a basis $\mathfrak{B}^{\prime}=\left\{w_{j}\right\}_{j \in J}$ of $W$ we say that a basis $\mathfrak{B}=\left\{v_{i}\right\}_{i \in I}$ of $V$ is multiplicative respect $\mathfrak{B}^{\prime}$ if for any $i \in I$ and $j \in J$ we have either $v_{i} w_{j}=0$ or $0 \neq v_{i} w_{j} \in \mathbb{F} v_{k}$ for some (unique) $k \in I$.

To construct examples of modules over linear spaces admitting a multiplicative basis, we just have to fix two non-empty sets $I, J$ and two arbitrary mappings $\alpha: I \times J \rightarrow$ $I$ and $\beta: I \times J \rightarrow \mathbb{F}$. Then the $\mathbb{F}$-linear space $V$ with basis $\mathfrak{B}=\left\{v_{i}\right\}_{i \in I}$ is a module respect to the $\mathbb{F}$-linear space $W$ with basis $\mathfrak{B}^{\prime}=\left\{w_{j}\right\}_{j \in J}$, under the action induced by $v_{i} w_{j}:=\beta(i, j) v_{\alpha(i, j)}$, admitting $\mathfrak{B}$ as multiplicative basis respect to $\mathfrak{B}^{\prime}$.

Remark 1. Definition 2 agree with the one for arbitrary algebras given in [5], and it is a little bit more general than the usual one in the literature $([1-4,10])$.

\section{Connections in the set of indexes. Decompositions}

From now on and throughout the paper, $V$ denotes a module over the linear space $W$, both endowed with respective basis $\mathfrak{B}=\left\{v_{i}\right\}_{i \in I}, \mathfrak{B}^{\prime}=\left\{w_{j}\right\}_{j \in J}$, and being $\mathfrak{B}$ multiplicative respect to $\mathfrak{B}^{\prime}$. We denote by $\mathcal{P}(I)$ the power set of $I$.

We begin this section by developing connection techniques among the elements in the set of indexes $I$ as the main tool in our study. For each $j \in J$, a new variable $\bar{j} \notin J$ is introduced and we denote by $\bar{J}:=\{\bar{j}: j \in J\}$ the set of all these new symbols. We will also write $\overline{(\bar{j})}:=j \in J$.

We consider the operation $\star: I \times(J \cup \bar{J}) \rightarrow \mathcal{P}(I)$ given by:

- If $i \in I$ and $j \in J$,

$$
i \star j:=\left\{\begin{array}{c}
\emptyset \text { if } 0=v_{i} w_{j} \\
\{k\} \text { if } 0 \neq v_{i} w_{j} \in \mathbb{F} v_{k}
\end{array}\right.
$$

- If $i \in I$ and $\bar{j} \in \bar{J}$,

$$
i \star \bar{j}:=\left\{k: 0 \neq v_{k} w_{j} \in \mathbb{F} v_{i}\right\}
$$

Now, we also consider the mapping $\phi: \mathcal{P}(I) \times(J \dot{\cup} \bar{J}) \rightarrow \mathcal{P}(I)$ defined as $\phi(U, j):=\bigcup_{i \in U}(i \star j)$.

Lemma 1. Let $a, b \in I$ be. Given $j \in J \dot{U} \bar{J}$ we have that $a \in b \star j$ if and only if $b \in a \star \bar{j}$.

Proof. Let us suppose that $a \in b \star j$. If $j \in J$ then $v_{b} w_{j} \in \mathbb{F} v_{a}$, and if $j \in \bar{I}$ we have $v_{a} w_{\bar{j}} \in \mathbb{F} v_{b}$. In any case $b \in a \star \bar{j}$. To prove the converse we can argue in a similar way.

Lemma 2. Given $j \in J \cup \bar{J}$ and $U \subset \mathcal{P}(I)$ then $i \in \phi(U, j)$ if and only if $\phi(\{i\}, \bar{j}) \cap$ $U \neq \emptyset$.

Proof. Let us suppose that $i \in \phi(U, j)$. Then there exists $k \in U$ such that $i \in k \star j$. By Lemma 1 we have $k \in i \star \bar{j}=\phi(\{i\}, \bar{j})$. So $k \in \phi(\{i\}, \bar{j}) \cap U \neq \emptyset$. By arguing in a similar way the converse can be proven. 
Definition 3. Let $i, k \in I$ be with $i \neq k$. We say that $i$ is connected to $k$ if there exists a subset $\left\{j_{1}, \ldots, j_{n}\right\} \subset J \cup \bar{J}$, such that the following conditions hold:

1. $\phi\left(\{i\}, j_{1}\right) \neq \emptyset, \phi\left(\phi\left(\{i\}, j_{1}\right), j_{2}\right) \neq \emptyset, \ldots, \phi\left(\phi\left(\ldots \phi\left(\{i\}, j_{2}\right) \ldots\right), j_{n-1}\right) \neq \emptyset$.

2. $k \in \phi\left(\phi\left(\ldots \phi\left(\{i\}, j_{1}\right) \ldots\right), j_{n}\right)$.

We say that $\left\{j_{1}, \ldots, j_{n}\right\}$ is a connection from $i$ to $k$ and we accept $i$ is connected to itself.

Lemma 3. Let $\left\{j_{1}, j_{2}, \ldots, j_{n-1}, j_{n}\right\}$ be any connection from some $i$ to some $k$ where $i, k \in I$ with $i \neq k$. Then the set $\left\{\bar{j}_{n}, \bar{j}_{n-1}, \ldots, \bar{j}_{2}, \bar{j}_{1}\right\}$ is a connection from $k$ to $i$.

Proof. Let us prove it by induction on $n$. For $n=1$ we have that $k \in \phi\left(\{i\}, j_{1}\right)$. It means that $k \in i \star j_{1}$ and so, by Lemma $1, i \in k \star \bar{j}_{1}=\phi\left(\{k\}, \bar{j}_{1}\right)$. Hence $\left\{\bar{j}_{1}\right\}$ is a connection from $k$ to $i$.

Let us suppose that the assertion holds for any connection with $n \geq 1$, elements and let us show this assertion also holds for any connection $\left\{j_{1}, j_{2}, \ldots, j_{n}, j_{n+1}\right\}$.

By denoting the set $U:=\phi\left(\phi\left(\ldots \phi\left(\{i\}, j_{1}\right) \ldots\right), j_{n}\right)$ and taking into the account the second condition of Definition 3 we have that $k \in \phi\left(U, j_{n+1}\right)$. Then, by Lemma 2, $\phi\left(\{k\}, \bar{j}_{n+1}\right) \cap U \neq \emptyset$ and so we can take $h \in U$ such that

$$
h \in \phi\left(\{k\}, \bar{j}_{n+1}\right) .
$$

Since $h \in U$ we have that $\left\{j_{1}, j_{2}, \ldots, j_{n-1}, j_{n}\right\}$ is a connection from $i$ to $h$. Hence $\left\{\bar{j}_{n}, \bar{j}_{n-1}, \ldots, \bar{j}_{2}, \bar{j}_{1}\right\}$ connects $h$ with $i$. From here and by Equation (1) we obtain $i \in \phi\left(\phi\left(\ldots \phi\left(\phi\left(\{k\}, \bar{j}_{n+1}\right), \bar{j}_{n}\right) \ldots\right), \bar{j}_{1}\right)$. So $\left\{\bar{j}_{n+1}, \ldots, \bar{j}_{2}, \bar{j}_{1}\right\}$ connects $k$ with $i$.

Proposition 1. The relation $\sim$ in $I$, defined by $i \sim k$ if and only if $i$ is connected to $k$, is an equivalence relation.

Proof. The reflexive and symmetric character is given by Definition 3 an Lemma 3.

If we consider the connections $\left\{a_{1}, \ldots, a_{m}\right\}$ and $\left\{b_{1}, \ldots, b_{n}\right\}$ from $a$ to $b$ and from $b$ to $c$ respectively, then is easy to prove that $\left\{a_{1}, \ldots, a_{m}, b_{1}, b_{2}, \ldots, b_{n}\right\}$ is a connection from $a$ to $c$. So $\sim$ is transitive and consequently an equivalence relation.

By the above Proposition we can introduce the quotient set $I / \sim:=\{[i]: i \in I\}$, becoming $[i]$ the set of elements in $I$ which are connected to $i$.

Recall that a submodule $Y$ of a module $V$ (respect to the linear space $W$ ) is a linear subspace of $V$ such that $Y W \subset Y$. Our next aim is to associate an (adequate) submodule to each $[i] \in I / \sim$. We define the linear subspace $V_{[i]}:=\bigoplus_{j \in[i]} \mathbb{F} v_{j}$.

Proposition 2. For any $i \in I / \sim$ we have that $V_{[i]}$ is a submodule of $V$.

Proof. We need to check $V_{[i]} W \subset V_{[i]}$. Suppose there exist $i_{1} \in[i], j_{1} \in[j]$ such that $0 \neq v_{i_{1}} w_{j_{1}} \in v_{n}$, for some $n \in I$. Therefore $n \in \phi\left(\left\{i_{1}\right\}, j_{1}\right)$. Considering the connection $\left\{j_{1}\right\}$ we get $i_{1} \sim n$, and by transitivity $n \in[i]$. 
Definition 4. We say that a submodule $Y \subset V$ admits a multiplicative basis $\mathfrak{B}_{Y}$ inherited from $\mathfrak{B}$ if $\mathfrak{B}_{Y} \subset \mathfrak{B}$.

Observe that any submodule $V_{[i]} \subset V$ admits an inherited basis $\mathfrak{B}_{[i]}:=\left\{v_{j}: j \in\right.$ $[i]\}$. So we can assert

Theorem 1. Let $V$ be a module admitting a multiplicative basis $\mathfrak{B}$ respect to a fixed basis of $W$. Then

$$
V=\bigoplus_{[i] \in I / \sim} V_{[i]},
$$

being any $V_{[i]} \subset V$ a submodule admitting a multiplicative basis $\mathfrak{B}_{[i]}$ inherited from $\mathfrak{B}$.

We recall that a module $V$ is simple if its only submodules are $\{0\}$ and $V$.

Corollary 1. If $V$ is simple then any couple of elements of I are connected.

Proof. The simplicity of $V$ applies to get that $V_{[i]}=V$ for some $[i] \in I / \sim$. Hence $[i]=I$ and so any couple of elements in $I$ are connected.

\section{The minimal components}

In this section we show that, under mild conditions, the decomposition of $V$ of Theorem 1 can be given by means of the family of its minimal submodules. We begin by introducing a concept of minimality for $V$ that agree with the one for algebras in [5].

Definition 5. A module $V$, (over a linear space $W$ ), admitting a multiplicative basis $\mathfrak{B}$ respect to fixed basis of $W$, is said to be minimal if its unique nonzero submodule admitting a multiplicative basis inherited from $\mathfrak{B}$ is $V$.

Let us also introduce the concept of $\star$-multiplicativity in the framework of modules over linear spaces in a similar way to the analogous one for arbitrary algebras (see [5] for these notions and examples).

Definition 6. We say that a module $V$ respect $W$ admits a $\star$-multiplicative basis $\mathfrak{B}=$ $\left\{v_{i}\right\}_{i \in I}$ respect to a fixed basis $\mathfrak{B}^{\prime}=\left\{l_{j}\right\}_{j \in J}$ of $W$, if it is multiplicative and given $a, b \in I$ such that $b \in a \star j$ for some $j \in J \cup \dot{\cup} \bar{J}$ then $v_{b} \in v_{a} W$.

Theorem 2. Let $V$ be a module respect $W$ admitting $a \star$-multiplicative basis $\mathfrak{B}=$ $\left\{v_{i}\right\}_{i \in I}$ respect to the basis $\mathfrak{B}^{\prime}=\left\{w_{j}\right\}_{j \in J}$ of $W$. Then $V$ is minimal if and only if the set of indexes I has all of its elements connected.

Proof. The first implication is similar to Corollary 1. To prove the converse, consider a nonzero submodule $Y \subset V$ admitting a multiplicative basis inherited from $\mathfrak{B}$. Then, for a certain $\emptyset \neq I_{Y} \subset I$, we can write $Y=\bigoplus_{i \in I_{Y}} \mathbb{F} v_{i}$. Fix some $i_{0} \in I_{Y}$ being then

$$
0 \neq v_{i_{0}} \in Y \text {. }
$$


Let us show by induction on $n$ that if $\left\{j_{1}, j_{2}, \ldots, j_{n}\right\}$ is any connection from $i_{0}$ to some $k \in I$ then for any $h \in \phi\left(\phi\left(\cdots \phi\left(\left\{i_{0}\right\}, j_{1}\right) \ldots\right), j_{n}\right)$ we have that $0 \neq v_{h} \in Y$.

In case $n=1$, we get $h \in \phi\left(\left\{i_{0}\right\}, j_{1}\right)$. Hence $h \in i_{0} \star j_{1}$, then, taking into account that $Y$ is a submodule of $V$, by $\star$-multiplicativity of $\mathfrak{B}$ and Equation (2) we obtain $v_{h} \in v_{i_{0}} W \subset Y$.

Suppose now the assertion holds for any connection $\left\{j_{1}, j_{2}, \ldots, j_{n}\right\}$ from $i_{0}$ to any $r \in I$ and consider some arbitrary connection $\left\{j_{1}, j_{2}, \ldots, j_{n}, j_{n+1}\right\}$ from $i_{0}$ to any $k \in I$. We know that for $x \in U$, where $U:=\phi\left(\phi\left(\cdots \phi\left(\left\{i_{0}\right\}, j_{1}\right) \cdots\right), j_{n}\right)$, the element

$$
0 \neq v_{x} \in Y \text {. }
$$

Taking into account that the fact $h \in \phi\left(\phi\left(\cdots \phi\left(\left\{i_{0}\right\}, j_{1}\right) \ldots\right), j_{n+1}\right)$ means $h \in$ $\phi\left(U, j_{n+1}\right)$, we have that $h \in x \star j_{n+1}$ for some $x \in U$. From here, the $\star$-multiplicativity of $\mathfrak{B}$ and Equation (3) allow us to get $v_{h} \in v_{x} W \subset Y$ as desired.

Since given any $k \in I$ we know that $i_{0}$ is connected to $k$, we can assert by the above observation that $\mathbb{F} v_{k} \subset Y$. We have shown $V=\bigoplus_{k \in I} \mathbb{F} v_{k} \subset Y$ and so $Y=V$.

Theorem 3. Let $V$ be a module, over the linear space $W$, admitting $a \star$-multiplicative basis $\mathfrak{B}$ respect to a fixed basis of $W$. Then $V=\bigoplus_{k} V_{k}$ is the direct sum of the family of its minimal submodules, each one admitting $a \star$-multiplicative basis inherited from $\mathfrak{B}$.

Proof. By Theorem 1 we have $V=\bigoplus_{[i] \in I / \sim} V_{[i]}$ is the direct sum of the submodules $V_{[i]}$. Now for any $V_{[i]}$ we have that $\mathfrak{B}_{[i]}$ is a $\star$-multiplicative basis where all of the elements of $[i]$ are connected. Applying Theorem 2 to any $V_{[i]}$ we have that the decomposition $V=\bigoplus_{[i] \in I / \sim} V_{[i]}$ satisfies the assertions of the theorem.

\section{References}

1. Bautista, R., Gabriel, P., Roiter, A.V. and Salmeron, L.: Representation-finite algebras and multiplicative basis. Invent. math. 81 (1985), 217-285.

2. Bovdi, V.: On a filtered multiplicative bases of group algebras. II. Algebr. Represent. Theory 6 (2003), no. 3, 353-368.

3. Bovdi, V., Grishkov, A. and Siciliano, S.: Filtered multiplicative bases of restricted enveloping algebras. Algebr. Represent. Theory 14 (2011), no. 4, 601-608.

4. Bovdi, V., Grishkov, A. and Siciliano, S.: On filtered multiplicative bases of some associative algebras. Algebr. Represent. Theory. DOI 10.1007/s10468-014-9494-7.

5. Calderón, A.J. and Navarro, F.J.: Arbitrary algebras with a multiplicative basis. Linear Algebra and its Applications. In press.

6. Chu, Y.J., Huang, F., Zheng, Z.J.: A commutant of $\beta \gamma$-system associated to the highest weight module $V_{4}$ of $\operatorname{sl}(2, \mathbb{C})$. J. Math. Phys. 51(9), 092301, 32 pp, (2010).

7. Dimitrov, I., Futorny, V., Penkov, I.: A reduction theorem for highest weight modules over toroidal Lie algebras. Comm. Math. Phys. 250(1), 47-63, (2004).

8. Grantcharov, D., Jung, J.H., Kang, S.J., Kim, M.: Highest weight modules over quantum queer superalgebra $U_{q}(\mathfrak{q}(n))$. Comm. Math. Phys. 296(3), 827-860, (2010). 
9. Iohara, K.: Unitarizable highest weight modules of the $N=2$ super Virasoro algebras: untwisted sectors. Lett. Math. Phys. 91(3), 289-305, (2010).

10. Kupisch, H. and Waschbusch, J.: On multiplicative basis in quasi-Frobenius algebras. Math. Z. 186, (1984), 401-405.

11. Liu, D., Gao, S., Zhu, L.: Classification of irreducible weight modules over $W$-algebra $W(2,2)$. J. Math. Phys. 49(1), 113503, 6 pp, (2008).

12. Takemura, K.: The decomposition of level-1 irreducible highest-weight modules with respect to the level-0 actions of the quantum affine algebra. J. Phys. A 31, n. 5., 1467-1485, (1998).

13. Zapletal, A.: Difference equations and highest-weight modules of $U_{q}[\operatorname{sl}(n)]$. J. Phys. A 31, n. 47, 9593-9600, (1998). 